\title{
Lung Abscess Remains a Life-Threatening Condition in Pediatrics - A Case Report
}

\author{
Ioana Raluca Chirteș¹, Cristina Oana Mărginean2,4*, Horea Gozar ${ }^{3,4}$, Anca Meda \\ Georgescu $^{1,4}$, Lorena Elena Meliț 2,4 \\ 1 Infectious Diseases Clinic 1 Tîrgu Mureș, Romania \\ 2 Paediatrics Clinic 1 Tîrgu Mureș, Romania \\ 3 Paediatric Surgery and Orthopedics Clinic Tîrgu Mureș, Romania \\ 4 University of Medicine and Pharmacy Tîrgu Mureș, Romania
}

\begin{abstract}
Pulmonary abscess or lung abscess is a lung infection which destroys the lung parenchyma leading to cavitations and central necrosis in localised areas formed by thick-walled purulent material. It can be primary or secondary. Lung abscesses can occur at any age, but it seems that paediatric pulmonary abscess morbidity is lower than in adults. We present the case of a one year and 5-month-old male child admitted to our clinic for fever, loss of appetite and an overall altered general status. Laboratory tests revealed elevated inflammatory biomarkers, leukocytosis with neutrophilia, anaemia, thrombocytosis, low serum iron concentration and increased lactate dehydrogenase level. Despite wide-spectrum antibiotic therapy, the patient's progress remained poor after seven days of treatment and a CT scan established the diagnosis of a large lung abscess. Despite changing the antibiotic therapy, surgical intervention was eventually needed. There was a slow but steady improvment and eventually, the patient was discharged after approximately five weeks.
\end{abstract}

Keywords: lung abscess, child, sepsis

Received: 5 June 2017 / Accepted: 25 July 2017

\section{INTRODUCTION}

Lower respiratory tract infections are a leading cause of death for approximately 3.9 million people per year worldwide, and of these, 1.8 million deaths occur in children under the age of five years [1]. Pulmonary abscess is an infectious condition which destroys the lung parenchyma leading to cavitations and central necrosis in localised areas, formed by thick-walled purulent material, and can primary or secondary [2]. Primary lung abscesses develop in previously healthy children without any known underlying conditions, being usually solitary, while secondary ones occur in children with underlying or predisposing disorders and these can be multiple [2]. Most frequently, lung abscess is a local complication of pneumonia. The clinical manifestations of lung abscess in children include a cough, fever, tachypnea, dyspnea, chest pain, vomiting, sputum pro- duction, weight loss and haemoptysis. The diagnosis is usually established by chest radiography or a CT scan in selected cases, the later being able to indicate the location, size and anatomic characteristics of a lesion [2].

The aetiology of lung abscess can be bacterial including (a) aerobic Streptococcus spp., Staphylococcus aureus, Escherichia coli, Klebsiella pneumonia, Pseudomonas aeruginosa, or very rarely Mycoplasma pneumoniae, (b) anaerobic such as Bacteroides spp., Fusobacterium spp., and Peptostreptococcus spp. or (c) fungal in immunocompromised patients [2]. Though lung abscess can occur at any age, it seems that paediatric pulmonary abscess morbidity is lower than in adults. Nevertheless, there are only a few reported studies reported regarding the prevalence and clinical outcome of this condition in children $[3,4]$. However the development of antimicrobial therapy over the last decades has improved the outcome of lung abscess, the 
data from 1969 to 2005 reported mortality rates, ranging from $2 \%$ to $38.2 \%$ [5].

The treatment of lung abscess can be conservative or surgical, with most physicians preferring conservative treatment as the treatment of choice. Conservative treatment involves parenteral antibiotics for two to three weeks, followed by a course of oral antibiotics for four to six weeks, concurrently with vital function support [2]. In severe cases, where there is little or no improvement after seven to ten days of therapy, surgical intervention is the appropriate therapeutic option [2].

\section{CASE REPORT}

\section{Medical history and presenting concerns}

The case is presented of a one year and 5-month-old male child admitted to Paediatric Clinic 1, Tg. Mures, with fever, loss of appetite and altered general status. Neither the family nor his personal history revealed any pathological elements. The onset of the disease occurred approximately ten days before admission, with a raised temperature of $39^{\circ} \mathrm{C}$ in spite of the administration of antipyretics. Therefore, the mother consulted a paediatrician who recommended antibiotic treatment (Cefuroxime suspension $125 \mathrm{mg} / 5 \mathrm{ml} ; 5 \mathrm{ml} 2$ times/ day). No improvement in the child's condition resulted and he was admitted to the territorial hospital where thoracic radiography revealed a massive right pneumonia with pleural effusion. The child was transferred to the Paediatrics Clinic $1 \mathrm{Tg}$. Mureș, Romania, for further investigations and treatment.

\section{Clinical findings}

The clinical examination on admission showed an altered general status, ringed face, pallor, cryptic and hyperaemic tonsils, hyperaemic pharynx, respiratory distress, abdominal breathing, vesicular murmur absent on the right lung, peripheral oxygen saturation $94 \%$, heart rate 134 beats/minute. The patient weighed 10 kilogrammes, and his height was 95 centimetres.

\section{Diagnostic focus and assessment}

The laboratory tests performed on the day of admission revealed a severe inflammatory syndrome with increased inflammatory biomarkers. C-reactive protein (CRP) $299 \mathrm{mg} / \mathrm{L}$, erythrocyte sedimentation rate (ESR) $130 \mathrm{~mm} / \mathrm{h}$; leukocytosis (Leu $24550 / \mu \mathrm{L}$ ) with neutrophilia (Neu $16680 / \mu \mathrm{L}$ ), thrombocytosis (Plt $883 / \mu \mathrm{L}$ ), anemia (Hb 7.3 g/dL, Htc 23.2\%, MEV 66.9fL, MEH $21 \mathrm{pg})$, low serum iron $(3.21 \mu \mathrm{mol} / \mathrm{l})$, and an increased level of lactate dehydrogenase (LDH 398 U/L).

A blood smear indicated 1\% myelocyte, 3\% nonsegmented cells, $69 \%$ segmented cells, $11 \%$ monocytes, $16 \%$ lymphocytes with hypochromic, microcytic red blood cells, increased number of platelets, hyper granulated and hyper segmented polymorphonuclear cells.

A blood culture was negative. A chest X-ray showed a massive right pneumonia with pleural effusions. (Fig. 1)

\section{Therapeutic focus and assessment}

Based on the clinical, laboratory and radiological findings a diagnosis was a diagnosis was made of staphylococcal pleural-pneumonia. Antibiotic treatment with meropenem (200 mg 3 times a daily for 7 days) and vancomycin (100 mg 4 times daily for 7 days) together with oxygen by mask during the first three days of admission, electrolytes (sodium chloride and potasium chloride) and glucose solutions by vein during the first week of admission depeding on the oral intake and symptomatic treatment (antipyretics - paracetamol $125 \mathrm{mg} /$ dose up to $600 \mathrm{mg} /$ day, inhaled bronchodilator therapy with salbutamol sulphate $5 \mathrm{mg} / \mathrm{ml} \quad 0,5 \mathrm{ml}$ up to $1 \mathrm{ml} /$ dose). We initiated the treatment with antipyretics followed by inhaled bronchodilator therapy with salbutamol and rehydration therapy. At the same time we administrated the above mentioned antibiotherapy.

Unfortunately, after one week of the above mentioned antibiotic treatment, the fever persisted, and the patient developed bilateral lower limbs oedema. Subsequent laboratory tests showed approximately the same level of inflammatory biomarkers (CRP $291 \mathrm{mg} / \mathrm{L}$, ESR $120 \mathrm{~mm} / \mathrm{h})$, decreasing level of $\mathrm{Hb}(6.1 \mathrm{~g} / \mathrm{dL})$, and low level of albumin and total proteins (Alb $2.14 \mathrm{~g} / \mathrm{dL}$, TP $5.05 \mathrm{~g} / \mathrm{dL}$ ) as previously recorded. A chest X-ray showed a massive fluid cavity with air inclusions in the upper and middle right lobes associated with right pleural effusion compressing the mediastinum. (Fig. 2)

A thoracic CT established the diagnosis of a massive lung abscess, partially evacuated, with a cranial-caudal diameter of approximately $74 \mathrm{~mm}$, and secluded pleural effusion. The paediatric surgeon refused surgical treatment based on the patient's relatively good general status without signs of respiratory distress. An infectious diseases specialist recommended the following antibiotic regimen: meropenem $200 \mathrm{mg} 3$ times daily, metronidazole $60 \mathrm{mg} 3$ times daily, amikacin $75 \mathrm{mg} 2$ times daily, and levofloxacin $50 \mathrm{mg} 2$ times daily for 


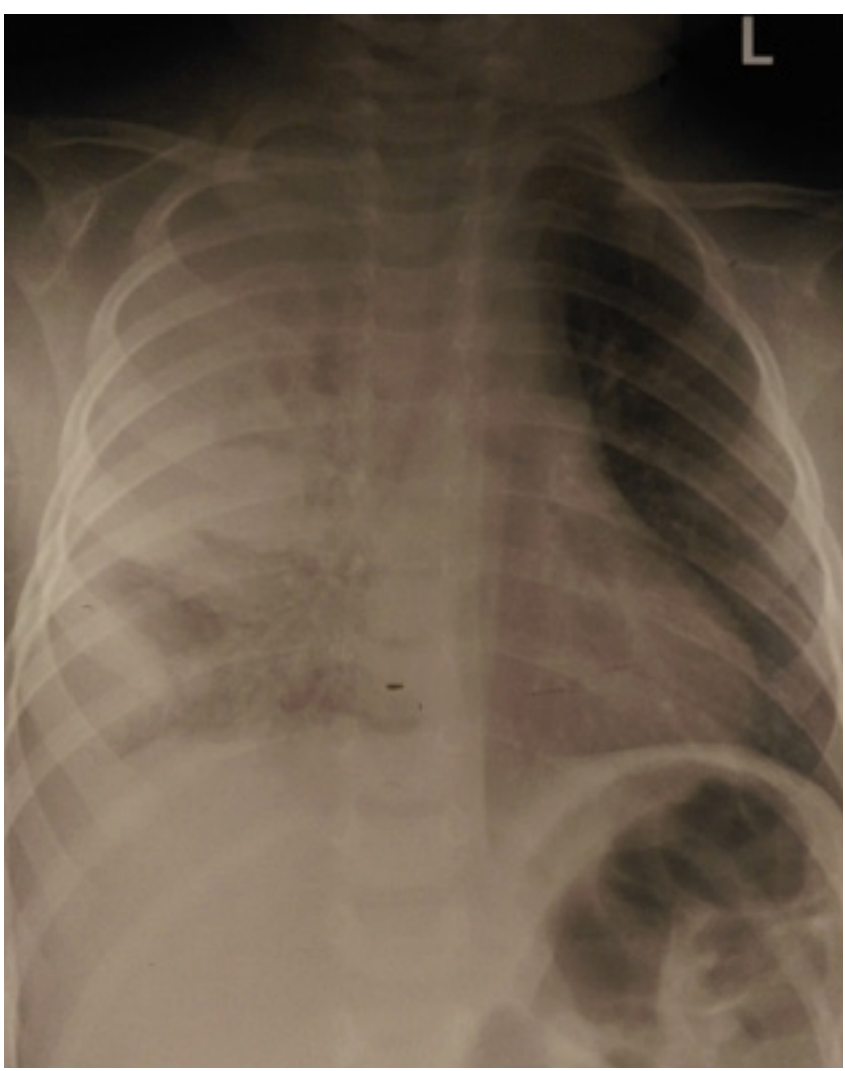

Fig. 1. Radiological aspect of the right lung on the day of admission

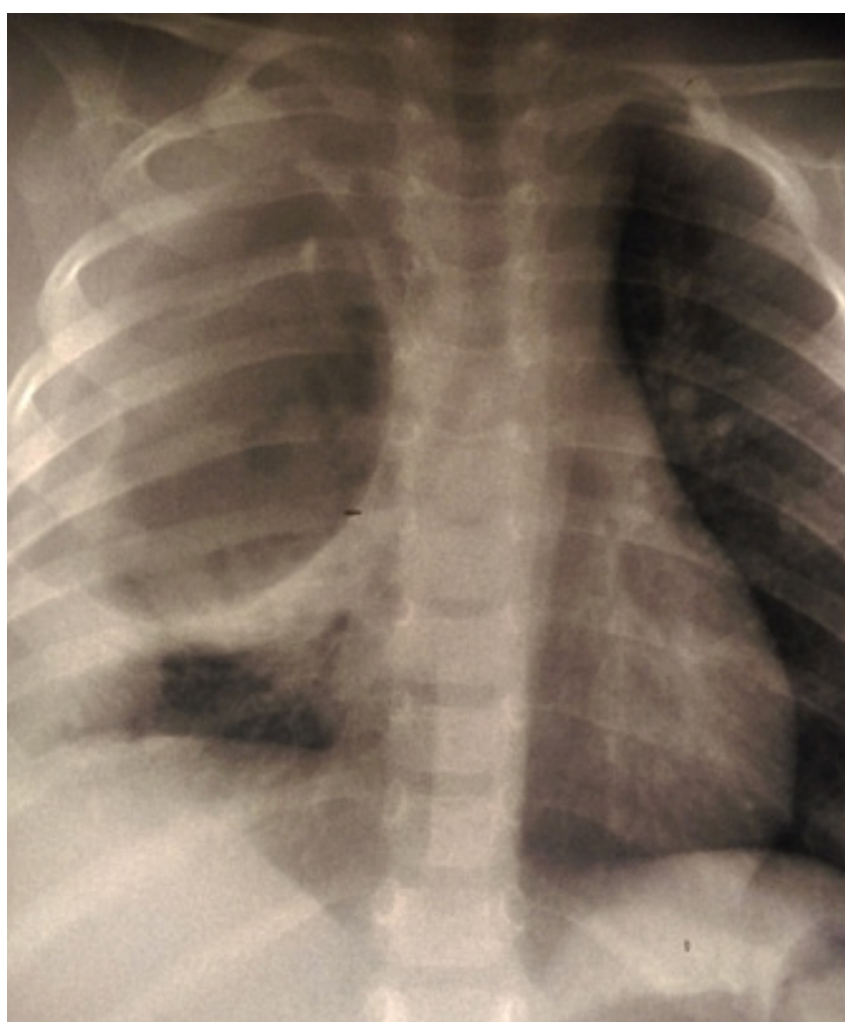

Fig. 2. Radiological aspect of the lung abscess one week. Human albumin $10 \mathrm{~g}$ per day for three days, blood transfusion $100 \mathrm{ml}$ once, and immunoglobulin $5 \mathrm{~g}$ a day for five days parenterally, were also administered, resulting in a favourable clinical evolution after three days of treatment.

Laboratory tests were repeated one week after the commencement of the new antibiotic regimen and showed that the inflammatory biomarkers remained elevated. A second CT was taken which revealed right fluid-pneumothorax compressing the mediastinum and causing the collapse of the right superior lobe, and an air cavity of approximately $49 \mathrm{~mm}$ diameter with a fluid content of approximately $19 \mathrm{~mm}$ axial diameter.

The patient was transferred to the Paediatric Surgery and Orthopedic Clinic Tg Mureș where a right pleurostomy was carried out. Afterwards, he was admitted to the Paediatric Intensive Care Unit for three days. A bronchoscopy indicated an atelectasis of the superior right lobe due to purulent secretions. The amount of drained purulent secretions was approximately 40-50 $\mathrm{ml}$ per day. A culture from the drained fluid, as well as a specific bacteriological culture, were both negative for Koch bacillus. Treatment was continued with meropenem $200 \mathrm{mg} 3$ times daily and levofloxacin $50 \mathrm{mg} 2$ times daily for another three weeks to cover both Gram positive and negative bacteria and anaerobic bacteria.

The pleurostomy was suppressed after two weeks, and the patient was discharged soon after.

\section{Discussion}

Lung abscess in children is very rare, accounting for approximately 0.7 per 100000 admissions per year [6] with a continuing decrease in their incidence due to major pharmacological developments. Most cases of paediatric lung abscess arise due to a complication of bacterial pneumonia [7]. There are, however, other predisposing factors that can lead to this condition in children such as immunodeficiency syndromes or immunosuppression states due to viral infections, severe systemic disorders and neurological conditions which can lead to aspiration lung disease [8].

Even though in the present case the lung abscess was the result of pneumonia, no predisposing factors or underlying conditions were identified that could have led to this condition. One of the most severe and common complications of lung abscess is sepsis, a critical condition with a wide spectrum of aetiologies, but which usually carries a good prognosis in children $[9,10]$. 
The most common aetiological agents of lung abscess in paediatric patients are Gram-positive cocci including Staphylococcus aureus and Streptococcus pneumonia and anaerobic bacteria. In immunocompromised or aspiration patients, several Gram-negative bacteria have been identified, such as Pseudomonas aeruginosa and Klebsiella pneumoniae [3]. Nevertheless, other rare aetiologies can be encountered such as that identified by Ruffini et al., (2014) who showed that a Mycoplasma pneumoniae infection might predispose to severe infections caused by typical respiratory pathogens [11].

Though we did not identify the precise aetiology in our case, it was most likely that the condition was caused by Gram-positive cocci, probably Staphylococcus aureus.

The diagnosis of lung abscess is usually established radiologically, but in certain cases, it can be difficult to differentiate between empyema and a lung abscess, based solely on a chest radiography. In such cases, it is necessary to perform a CT scan to confirm a diagnosis [12].

The first step in the management of a lung abscess is by parenteral antibiotics together with physiotherapy to facilitate postural drainage [13]. In most cases, due to the difficulties in establishing the aetiology in paediatric patients, antibiotic therapy is administered empirically [14].

Surgical drainage or percutaneous drainage should be limited to those cases which are refractory to medical therapy or who develop complications like bronchopleural fistula [15].

Our case was refractory to medical therapy and developed a right fluid-pneumothorax compressing the mediastinum and collapse of the right superior lobe. It, therefore, required surgical drainage. The consensus opinion is that in cases of children under the age of seven years, lung abscess does not respond readliy to medical therapy and does not drain spontaneously [13], and it follows that up to $21 \%$ of patients diagnosed with lung abscess, who do not respond to antibiotics, are likely to need surgical or percutaneous drainage [16]. Even though lung abscesses in paediatric patients are rare conditions, our case proves that they can develop despite the early administration of wide-spectrum antibiotics in children without any predisposing factors or underlying conditions, probably due to the bacterial strain involved and its pathogenicity.

\section{- CONCLUSIONS}

Lung abscess infrequently occurs in paediatric patients and is commonly a complication of bacterial pneumonia. Despite the fact that we initiated wide-spectrum antibiotics and vital functional support from the first day of admission, our patient developed a massive lung abscess with multiple complications eventually requiring surgical drainage. The eventual outcome and prognosis for the patient were favourable.

\section{CONFLICT OF INTEREST}

None to declare.

\section{ABbreviations}

Alb - albumin, CRP - C-reactive protein, ESR - erythrocyte sedimentation rate, $\mathrm{Hb}$ - haemoglobin, Htc - hematocrit, $\mathrm{MEH}$ - medium erythrocyte haemoglobin, MEV - medium erythrocyte volume, LDH - lactate dehydrogenase, Leu - leukocytes, Neu - neutrophils, Plt - platelets, TP - total proteins

\section{DEFERENCES}

1. GBD_report_2004update_full.pdf [Internet]. [cited 2017 Jul 22]. Available from: http://www.who.int/healthinfo/global_ burden_disease/GBD_report_2004update_full.pdf

2. Lasker OJ. Pulmonary abscess. In: Kliegman RM, Stanton BF, St Geme III JW, Schor NF: Nelson Texbook of Paediatrics. 20th ed. Elsevier; 2016. p. 2096-7.

3. Patradoon-Ho P, Fitzgerald DA. Lung abscess in children. Paediatr Respir Rev. 2007;8(1):77-84.

4. Miller MA, Ben-Ami T, Daum RS. Bacterial pneumonia in neonates and older children. In: Taussig LM, Landau LI, editors Paediatric respiratory medicine. St Louis: Mosby; 1999. p. 5448.

5. Takayanagi N, Kagiyama N, Ishiguro T, Tokunaga D, Sugita $Y$. Aetiology and outcome of community-acquired lung abscess. Respir Int Rev Thorac Dis. 2010;80(2):98-105.

6. Tan TQ, Seilheimer DK, Kaplan SL. Paediatric lung abscess: clinical management and outcome. Pediatr Infect Dis J. 1995;14(1):51-5.

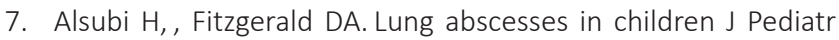
Inf Dis 2009; 4:27-35.

8. de Benedictis FM, Carnielli VP, de Benedictis D. Aspiration lung disease. Pediatr Clin North Am. 2009; 56:173-190, xi.

9. Melit Lorena Elena, Marginean Cristina Oana, Georgescu A, Duicu C. Complications of sepsis in infant. A case report. J Crit 
Available online at: www.jccm.ro

Care Med. 2016;2(2):96-9.

10. Grama Alina, Marginean Cristina Oana, Melit Lorena Elena, Georgescu A. Staphylococcal Scalded Skin Syndrome in Child. A Case Report and a Review from Literature. J Crit Care Med. 2016;2(4):192-7.

11. Ruffini E, De Petris L, Candelotti P, Tulli M, Sabatini MR, Luciani $L$, et al. Lung abscess in a child secondary to Mycoplasma pneumoniae infection. Pediatr Medica E Chir Med Surg Pediatr. 2014; 36:87-9.

12. Kuhlman JE, Singha NK. Complex disease of the pleural space: radiographic and CT evaluation. Radiogr Rev Publ Radiol Soc N Am Inc. 1997;17(1):63-79.
The Journal of Critical Care Medicine 2017;3(3) • 127

13. Reynolds JH, McDonald G, Alton H, Gordon SB. Pneumonia in the immunocompetent patient. Br J Radiol. 2010;83(996):9981009.

14. Emanuel B, Shulman ST. Lung abscess in infants and children. Clin Pediatr (Phila). 1995;34(1):2-6.

15. Chan P-C, Huang L-M, Wu P-S, Chang P-Y, Yang T-T, Lu C-Y, et al. Clinical management and outcome of childhood lung abscess: a 16-year experience. J Microbiol Immunol Infect Wei Mian Yu Gan Ran Za Zhi. 2005;38(3):183-8.

16. Erasmus JJ, McAdams HP, Rossi S, Kelley MJ. Percutaneous management of intrapulmonary air and fluid collections. Radiol Clin North Am. 2000;38(2):385-93. 